\title{
A46 THE EXPRESSION REGULATION OF THE HSPA1B GENE IN PATIENTS WITH MYOSITIS IS NOT DEPENDENT ON THE PRESENCE OF HLA-DRB1*03 RISK ALLELE
}

Martina Remakova, ${ }^{1}$ Marek Skoda, ${ }^{1}$ Marketa Faustova, ${ }^{1}$ Herman Mann, ${ }^{2}$ Olga Krystufková, ${ }^{3}$ Jiri Vencovsky, ${ }^{4}$ Peter Novota ${ }^{1}$ Department of Molecular Biology and Immunogenetics, Scientific Research Center, Institute of Rheumatology, Prague, Czech Republic; ${ }^{2}$ Clinical Department, Institute of Rheumatology, Prague, Czech Republic; ${ }^{3}$ Department of Flow Cytometry, Scientific Research Center, Institute of Rheumatology, Prague, Czech Republic; ${ }^{4}$ Department of Rheumatology, Scientific Research Center, Institute of Rheumatology, Prague, Czech republic

\subsection{6/ard.2010.148965.16}

Background and objectives Genes within the major histocompatibility complex (MHC) region has a strong genetic relevance in autoimmunity development. The involvement of the class I and class II genes, as well as class III (nonClass I/II MHC) genes has been proposed. For the myositis development, the HLA-DRB1*03 is a known risk factor in Caucasian population. However, there are another genes appearing to be significant players in the ethiology of myositis located near the HLA-DRB1 locus. In the present study the authors have focused on one of these risk factors - the regulation of MHC-linked inducible HSP7O genes expression and their relation to the known immunogenetic risk factor located within the MHC (HLA-DRB1*03).

Materials and methods The authors have investigated the gene specific HSP70 expression in 20 patients with dermatopolymyositis and 15 healthy people matching in age as control samples. Expression levels of the two inducible HSP7O genes (HSPA1A, HSPA1B) were analysed both in patients and controls. Both of the groups were additionally genotyped for HLA-DRB1 locus. Myositis-specific and associated autoantibodies were also identified in patients.

Results The expression of both, the HSPA1A and HSPA1B genes was significantly upregulated $(\mathrm{p}<0.001 ; \mathrm{p}<0.05)$ in patients suffering from myositis when compared to controls. The expression regulation of the $H S P A 1 A$ was found to be associated with the presence of the HLA-DRB1*03 risk allele in patients. However, this was not observed for the HSPA1B gene. In contrast, the authors found a positive correlation between the expression regulation of the HSPA1B gene and the presence of disease specific autoantibodies in myositis patients. Additionally, positive correlation between the presence of disease specific autoantibodies and the HLA-DRB1*03 risk allele was found. None of these observations were found in healthy controls. 
Conclusions The results suggest that the two MHC-linked inducible genes are differentially expressed in dependence on the autoantibody or HLA risk allele presence. The differential gene expression regulation shows that the HSPA1B is an - on HLA-DRB1 - independent molecular marker for myositis development. 